\title{
CRISPR-PCDup: a novel approach for simultaneous segmental chromosomal duplication in Saccharomyces cerevisiae
}

\author{
Naim Hassan ${ }^{1 \dagger}$, Yu Sasano ${ }^{1 \dagger}$, Shunta Kimura ${ }^{2}$, Farhana Easmin ${ }^{1}$, Keisuke Ekino ${ }^{1}$, Hisataka Taguchi ${ }^{1}$ \\ and Satoshi Harashima ${ }^{*^{*}}$ (1)
}

\begin{abstract}
In our previous study, a novel genome engineering technology, PCR-mediated chromosome duplication (PCDup), was developed in Saccharomyces cerevisiae that enabled the duplication of any desired chromosomal region, resulting in a segmental aneuploid. From one round of transformation, PCDup can duplicate a single chromosomal region efficiently. However, simultaneous duplication of multiple chromosomal regions is not possible using PCDup technology, which is a serious drawback. Sequential duplication is possible, but this approach requires significantly more time and effort. Because PCDup depends upon homologous recombination, we reasoned that it might be possible to simultaneously create duplications of multiple chromosomal regions if we could increase the frequency of these events. Double-strand breaks have been shown to increase the frequency of homologous recombination around the break point. Thus, we aimed to integrate the genome editing tool CRISPR/Cas9 system, which induces double-strand breaks, with our conventional PCDup. The new method, which we named CRISPR-PCDup increased the efficiency of a single duplication by up to 30 fold. CRISPR-PCDup enabled the simultaneous duplication of long chromosomal segments (160 kb and $200 \mathrm{~kb}$ regions). Moreover, we were also able to increase the length of the duplicated chromosome by up to at least $400 \mathrm{~kb}$, whereas conventional PCDup can duplicate up to a maximum of $300 \mathrm{~kb}$. Given the enhanced efficiency of chromosomal segmental duplication and the saving in both labor and time, we propose that CRISPR-PCDup will be an invaluable technology for generating novel yeast strains with desirable traits for specific industrial applications and for investigating genome function in segmental aneuploid.
\end{abstract}

Keywords: CRISPR/Cas9, Chromosomal Duplication, Homologous Recombination, Yeast

\section{Introduction}

Saccharomyces cerevisiae is a model organism of immense industrial interest. It is known that many of the characteristics essential for the industrial application of S. cerevisiae, such as stress tolerance, are controlled by more than one gene (Swinnen et al. 2012). Consequently, genome engineering technologies are required for the rapid and

\footnotetext{
*Correspondence: harashima@bio.sojo-u.ac.jp

${ }^{\dagger}$ Naim Hassan and Yu Sasano contributed equally to this work

1 Department of Applied Microbial Technology, Faculty of Biotechnology and Life Science, Sojo University, Ikeda 4-22-1, Nishi-ku, Kumamoto 860-0082, Japan

Full list of author information is available at the end of the article
}

effective exploitation of multiple genetic loci. Among various technologies, chromosome engineering is promising because it facilitates large scale genomic manipulation by altering chromosomes, thereby offering a powerful means of elucidating chromosome and genome function. Additionally, chromosome engineering can be used to generate useful yeast strains through the creation of a wide array of genetic diversity followed by a screening procedure to isolate the desired strains under defined culture conditions. However, a major limitation of chromosome engineering is the simultaneous manipulation of multiple chromosomal sites and regions. Previously, we developed a variety of new chromosome engineering technologies
Springer Open
C The Author(s) 2020. This article is licensed under a Creative Commons Attribution 4.0 International License, which permits use, sharing, adaptation, distribution and reproduction in any medium or format, as long as you give appropriate credit to the original author(s) and the source, provide a link to the Creative Commons licence, and indicate if changes were made. The images or other third party material in this article are included in the article's Creative Commons licence, unless indicated otherwise in a credit line to the material. If material is not included in the article's Creative Commons licence and your intended use is not permitted by statutory regulation or exceeds the permitted use, you will need to obtain permission directly from the copyright holder. To view a copy of this licence, visit http://creativeco mmons.org/licenses/by/4.0/. 
in S. cerevisiae. One such method, named PCR-mediated chromosome duplication (PCDup), enables the duplication of any desired chromosomal region as an independent chromosome (Natesuntorn et al. 2015). PCDup is able to duplicate chromosomal regions with lengths from $50 \mathrm{~kb}$ to $300 \mathrm{~kb}$. Using PCDup, we discovered that segmental duplication of some chromosome regions leads to an enhanced resistant phenotype when the cells are grown under stress conditions. However, the PCDup method has limitations because duplication is restricted to a single region at each transformation step. Simultaneous duplication of two or more target regions in the genome of an organism, even in the yeast genome, has not been achieved. Time is also a major consideration when conducting genome engineering. For example, one round of duplication takes at least 11 days including confirmatory analysis and if the results is in failure, constructing stains by successive multiple chromosome duplications is both time consuming and laborious.

PCDup technology is based on the mechanism of homologous recombination. We reasoned that an improvement of homologous recombination activity might be the key to enhancing chromosome duplication efficiency. It has previously been shown that induction of double-strand breaks (DSBs) can increase recombination efficiency near the site of the DSB by as much as 4000fold (Storici et al. 2003). Recently, RNA-guided programmable CRISPR/Cas systems have played a major role in facilitating precision genome engineering by sequencespecific introduction of double-strand breaks (DSBs) (Cong et al. 2013; Jinek et al. 2012; Sander and Joung 2014). Moreover, the CRISPR/Cas9 system has been shown to be functional in S. cerevisiae (DiCarlo et al. 2013). Thus, this method permits induction of site specific DSBs using an appropriate guide RNA (gRNA).

In this study, we introduced DSBs into the genome of $S$. cerevisiae using the CRISPR/Cas9 system before attempting a chromosome duplication. We show that the integration of CRISPR/Cas9 into our PCDup system, which we called CRISPR-PCDup, produces an effective genome engineering technology that enhances chromosomal duplication efficiency with a high level of fidelity and is capable of simultaneously targeting multiple chromosomal regions.

\section{Materials and Methods}

\section{Strains and media}

Strains used in this study are listed in Table 1. FY833 and FY834 cells containing plasmid p414-TEF1p-Cas9CYC1t were used as a host strain (SJY415 and SJY30, respectively) for the CRISPR-PCDup experiments. Escherichia coli DH5 $\alpha$ was used for plasmid construction and propagation. E. coli recombinant strains were grown in Luria-Bertani (LB) medium containing $100 \mu \mathrm{g} / \mathrm{ml}$ ampicillin. Yeast cells are grown in YPDA medium containing 1\% Bacto-Yeast Extract (BD Bioscience, San Jose, CA), 2\% Bacto-Peptone (Difco, Detroit, MI), 2\% glucose (Wako, Tokyo, Japan), $2 \%$ agar (Wako) and $0.004 \%$ adenine sulfate (Wako) and in Synthetic Complete (SC) medium containing $0.67 \%$ Yeast Nitrogen Base without Amino Acids (Difco), $0.2 \%$ dropout mix of amino acids and nucleic acid bases and 2\% glucose. SC medium lacking specific amino acids was used for the selection of transformants. For sporulation, diploid strain was cultivated

Table 1 Strains and plasmids used in the CRISPR-PCDup experiments

\begin{tabular}{|c|c|c|}
\hline Strain or plasmid & Description & Remarks \\
\hline \multicolumn{3}{|l|}{ Strain } \\
\hline FY833 & MATa ura3-52 his3- $\Delta 200$ leu2 $\Delta 1$ lys2 $2202 \operatorname{trp} 1 \Delta 63$ & (Winston et al. 1995) \\
\hline SJY415 & Trp+ transformants of FY833 harboring plasmid p414-TEF1p-Cas9-CYC1t & This study \\
\hline FY834 & MATa ura3-52 his3- $\Delta 200$ leu2 $\triangle 1$ lys2 $\triangle 202 \operatorname{trp} 1 \Delta 63$ & (Winston et al. 1995) \\
\hline SJY30 & Trp+ transformants of FY834 harboring plasmid p414-TEF1p-Cas9-CYC1t & (Sasano et al. 2016) \\
\hline \multicolumn{3}{|l|}{ Plasmid } \\
\hline pUG6 & $\begin{array}{l}\text { Containing loxP-flanked marker gene deletion cassette: loxP-pAgTEF1-kanMX-tAgTEF1- } \\
\text { loxP }\end{array}$ & (Güldener et al. 1996) \\
\hline p3009 & The loxP-CgHIS3-IoxP module containing plasmid constructed by modifying pUG6 & (Sugiyama et al. 2005) \\
\hline p3121 & The CEN4 module containing plasmid constructed by modifying pUG6 & (Sugiyama et al. 2005) \\
\hline p3122 & The CEN4-IoxP-CgLEU2-IoxP module containing plasmid constructed by modifying pUG6 & (Sugiyama et al. 2008) \\
\hline p3123 & The CEN4-loxP-CgHIS3-loxP module containing plasmid constructed by modifying pUG6 & (Sugiyama et al. 2008) \\
\hline pSJ23 & A derivative of pUG6 carrying URA3 & (Easmin et al. 2019b) \\
\hline pSJ69 & loxP site-deleted p3008 & (Easmin et al. 2019a) \\
\hline pSJ70 & loxP site deleted p3009 & (Easmin et al. 2019b) \\
\hline p414-TEF1p-Cas9- CYC1t & TEF1p-Cas9-CYC1t module containing YCp type plasmid & (DiCarlo et al. 2013) \\
\hline p426-SNR52p-gRNA.CAN1.Y-SUP4t & SNR52p-gRNA.CAN1 Y-SUP4t module containing YEp type plasmid & (DiCarlo et al. 2013) \\
\hline
\end{tabular}


in sporulation medium containing $1 \%$ potassium acetate, $0.1 \%$ bacto-yeast extract and $0.05 \%$ glucose. Agar $(2 \%$ $\mathrm{w} / \mathrm{v}$ ) was included for solid medium.

\section{CRISPR-PCDup}

Details of the conventional PCDup technology for chromosome duplication has been described previously (Natesuntorn et al. 2015). Briefly, two DNA modules necessary for duplication were prepared as follows. Each DNA module has $50 \mathrm{bp}$ homology sequence with the target and additionally contains either a selective marker (CgLEU2 or CgHIS3 or URA3) along with a telomere seed sequence (six copies of a $5^{\prime}$-CCCCAA-3') or a centromere along with or without selective marker (CgLEU2 or CgHIS3) and telomere seed sequence. p3121 (Sugiyama et al. 2005) was used as a template to add only centromere. p3122 (Sugiyama et al. 2008) was used to add centromere along with CgLEU2. p3123 (Sugiyama et al. 2008) was used to add centromere along with CgHIS3 to the module. p3009 (Sugiyama et al. 2005) was used as a template to prepare the duplication module containing CgHIS3. CEN4 sequence was added to one of the DNA modules so that the resulting new chromosomes possessed one centromere. Template plasmids used for targeting chromosomal regions are listed in Table 1. Primers for constructing the DNA module are listed in Additional file 1: Table S1. gRNA expression plasmids were constructed according to Sasano et al. (2016) and the software CRISPRdirect (https://crispr.dbcls .jp/) was used to select the $20 \mathrm{bp}$ target sequences. Oligonucleotide primers used for construction of gRNA expression plasmids are listed in Additional file 1: Table S2. For targeting each chromosomal region, two gRNA expressing plasmids (Sasano et al. 2016) were introduced (7.5 $\mu$ g each) along with the corresponding duplicating DNA modules into the transformation mixture. An outline of the CRISPR-PCDup method is shown in Fig. 1.

\section{Yeast and E. coli Transformation}

S. cerevisiae was transformed using the lithium acetate method (Gietz and Schiestl 2007). After transformation,

Selective marker (CgLEU2, CgHIS3)

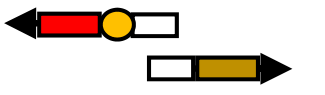

Duplicating modules

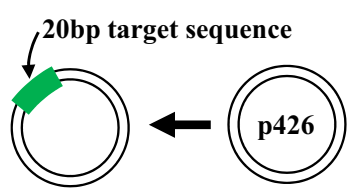

gRNA expressing plasmid

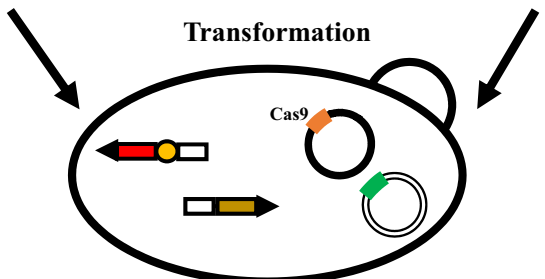

Host: SJY30
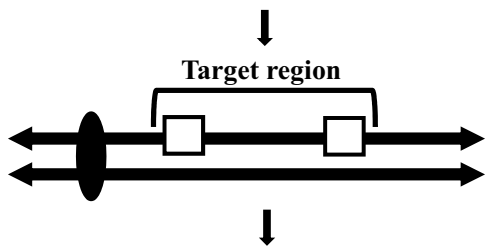

Double strand break by CRISPR/Cas9

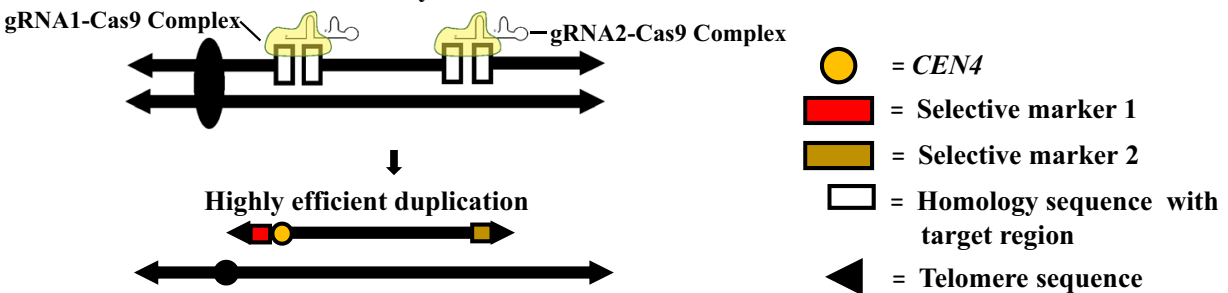

Fig. 1 Outline of the CRISPR-PCDup method. gRNA expressing plasmid and duplicating modules are introduced into the SJY30 strain, which harbors a Cas9-expressing plasmid. In transformed cells, CRISPR/Cas9 mediated double-strand breaks (DSBs) are induced near the target site. Chromosome duplication is then facilitated by PCDup. This new technology is named CRISPR-PCDup 
SC medium lacking the appropriate amino acids was used for selection of transformants having the marker gene from the duplicating module. E. coli was transformed according to the method described by Easmin et al. (2019a).

\section{Colony PCR, pulse field gel electrophoresis (PFGE) and Southern hybridization}

Colony PCR was performed according to the method described by Easmin et al. (2019a). Pulse field gel electrophoresis and Southern hybridization were performed according to Sasano et al. (2016). The oligonucleotide primers used for amplifying DNA fragments for probes in Southern hybridization are shown in Additional file 1: Table S3.

\section{Tetrad analysis}

Tetrad analysis was done according to Sugiyama et al. (2006).

\section{Results}

Increasing duplication efficiency by CRISPR-PCDup

We previously developed PCDup technology, which allows duplication of any desired chromosomal region of the S. cerevisiae genome (Natesuntorn et al. 2015). In this study, we developed CRISPR-PCDup technology, which is an integration of PCDup with CRISPR/Cas9 that facilitates simultaneous and multiple duplication of chromosome segments in S. cerevisiae. We reasoned that integration of the CRISPR/Cas9 system with PCDup might increase the frequency of homologous recombination, thereby enabling simultaneous duplication. Initially, we examined whether the CRISPR-PCDup method works more efficiently compared with the previous PCDup technology.

Cas9-expressing strain (SJY30) was used as host strain for chromosome segmental duplication. SJY30 showed no significant growth defect, which suggested that Cas9 expression is not toxic in this strain. We designed gRNA targeting sequences located just outside of the duplicating region and near both edges. The genomic positions chosen for duplication in this study and the gRNA targeting sequences are shown in Table 2 and Additional file 1: Table S4, respectively. Initially we attempted to produce duplication of the Chr3-1 region (1-158020) and Chr3-2 region (157543316620) on Chromosome 3 separately (Fig. 2). SJY30 strain was transformed with gRNA expressing plasmids and two kinds of duplication modules marked with CgHIS3 for Chr3-1 and CEN4+CgHIS3 for Chr3-2. Target sequences on these gRNA-expressing plasmids were located near the edge of the Chr3-1 and Chr3-2 regions (Additional file 1: Table S4). When the CRISPRPCDup system was employed, a total of 62 and 1316 $\mathrm{His}^{+}$transformants were obtained for the Chr3-1 and Chr3-2 region, respectively (Table 3). By contrast, using 50 bp homology sequence with the target, conventional PCDup gave only 2 and $51 \mathrm{His}^{+}$transformants under the same transformation conditions for the Chr3-1 and Chr3-2 region, respectively. We chose 8 transformants at random from those obtained by CRISPR-PCDup for the Chr3-1 and Chr3-2 region and subjected them to pulse field gel electrophoresis (PFGE) and subsequent Southern blot analysis to determine whether the duplication event had occurred at the expected locus. The

Table 2 Duplication of various chromosomal regions

\begin{tabular}{|c|c|c|c|}
\hline Duplication event & $\begin{array}{l}\text { Name of target } \\
\text { regions }\end{array}$ & $\begin{array}{l}\text { Size and coordinate number of the } \\
\text { target region }\end{array}$ & $\begin{array}{l}\text { Plasmids used for preparation } \\
\text { of the duplicating module }\end{array}$ \\
\hline \multirow[t]{8}{*}{ Single duplication } & Chr3-1 & Chr3 (1 - 158020) (158 kb) & p3009 \\
\hline & Chr3-2 & Chr3 (157543 - 316620) (160 kb) & p3123 \\
\hline & Chr5-3 & Chr5 (398496-576874) (177 kb) & pSJ70 \\
\hline & Chr15-L1 & Chr15 (569775 - 969009) (400 kb) & p3009, p3122 \\
\hline & Chr15-L2 & Chr15 (618914 - 969009) (350 kb) & p3009, p3122 \\
\hline & Chr15-L3 & Chr15 (670548 - 969009) (300 kb) & p3009, p3122 \\
\hline & Chr15-L4 & Chr15 (718509 - 969009) (250 kb) & p3009, p3122 \\
\hline & Chr15-L5 & Chr15 (767986 - 969009) (200 kb) & p3009, p3122 \\
\hline $\begin{array}{l}\text { Simultaneous double } \\
\text { duplication }\end{array}$ & Chr3-2 and Chr15-L5 & $\begin{array}{l}\text { Chr3 }(157543-316620)(160 \mathrm{~kb}) \text { and } \\
\text { Chr15 (767986 - 969009) (200 kb) }\end{array}$ & p3122 and p3009, p3121 \\
\hline \multirow{3}{*}{$\begin{array}{l}\text { Sequential } \\
\text { duplication }\end{array}$} & Chr3-1 & Chr3 (1 - 158020) (158 kb) & pSJ69 \\
\hline & Chr8-1 & Chr8 (1-202241) (200 kb) & pSJ70 \\
\hline & Chr14-4 & Chr14-4 (597394-784333) & pSJ23 \\
\hline
\end{tabular}




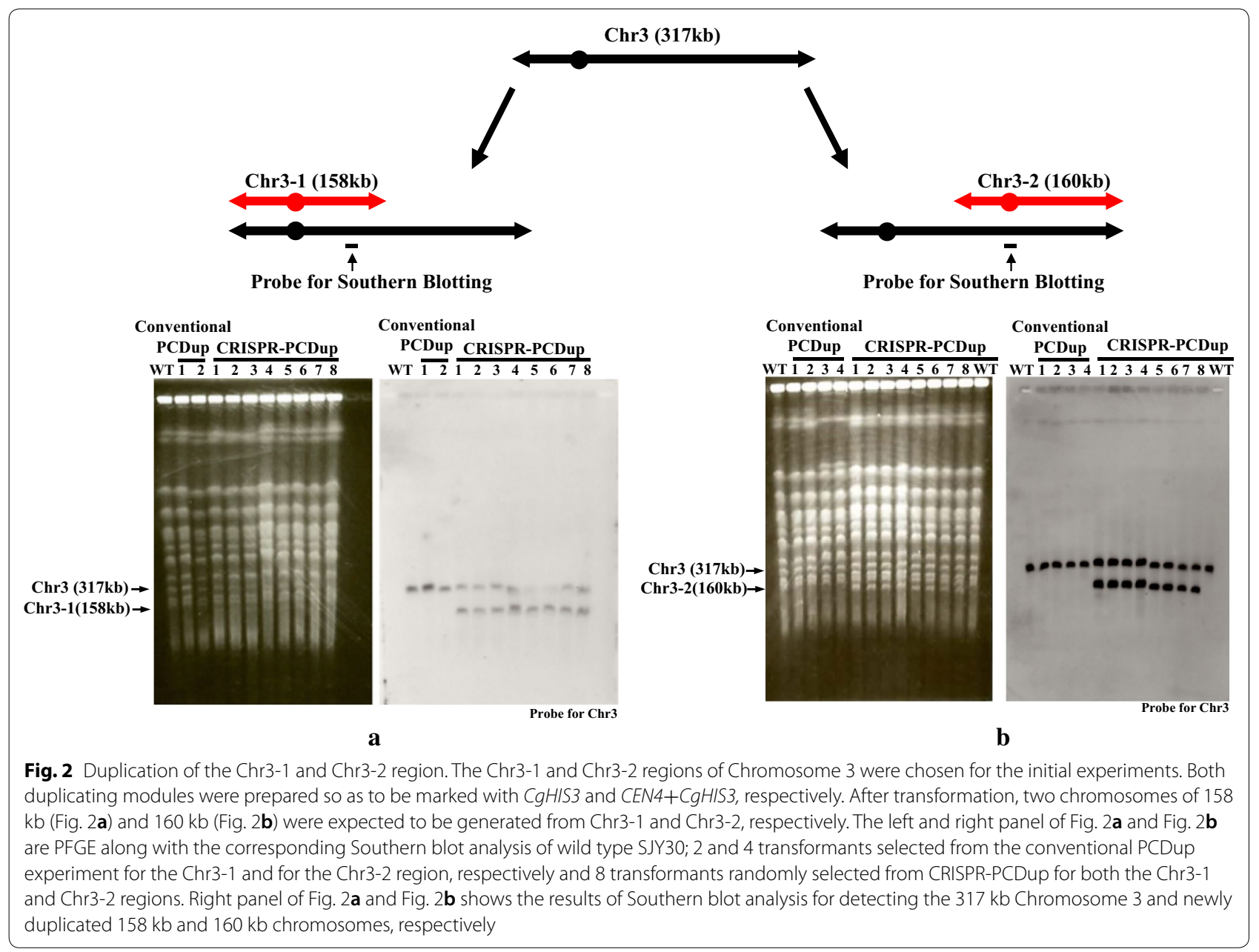

Table 3 CRISPR-PCDup increases duplication efficiency and induces simultaneous double duplication

\begin{tabular}{llccc}
\hline $\begin{array}{l}\text { Duplicated } \\
\text { regions }\end{array}$ & Method & Number of transformants $(\mathbf{n})$ & $\begin{array}{l}\text { Karyotype analysis } \\
(\mathbf{n})\end{array}$ & $\begin{array}{l}\text { Correct } \\
\text { transformants (n) }\end{array}$ \\
\hline Chr3-1 (158 kb) & CRISPR-PCDup & 62 & 8 & $8(100 \%)$ \\
& Conventional PCDup & 2 & 2 & $0(0 \%)$ \\
Chr3-2 (160 kb) & CRISPR-PCDup & 1316 & 8 & $8(100 \%)$ \\
& Conventional PCDup & 51 & 4 & $0(0 \%)$ \\
Chr3-2 (160 kb) and & CRISPR-PCDup & 75 & 25 & $10(40 \%)$ \\
Chr15-L5 (200 kb) & Conventional PCDup & 0 & - & - \\
\hline
\end{tabular}

Symbol "-" indicates that karyotype analysis and counting the number of correct transformants were not applicable

results of this analysis showed that all 8 transformants for duplication of the Chr3-1 and Chr3-2 regions had the expected duplicated chromosome (Fig. 2; i.e. $158 \mathrm{~kb}$ band in panel A and $160 \mathrm{~kb}$ band in panel B) in addition to intact Chromosome 3 (317 kb band). However, using conventional PCDup, 2 and 4 transformants were analyzed for the Chr3-1 and Chr3-2 region, respectively, but none had the expected duplicated chromosome (Fig. 2a, b). Based on these observations, we concluded that CRISPR-PCDup efficiently enhanced segmental duplication of a single chromosomal region.

\section{Simultaneous double duplication by CRISPR-PCDup}

Despite numerous attempts, simultaneous duplication of two different genomic regions by conventional PCDup has never been achieved. The results in the previous section revealed that the duplication of a single chromosomal region was possible at high frequency. Next, 
we attempted to induce a simultaneous duplication of two genomic regions on different chromosomes, namely Chr3-2 (160 kb) and Chr15-L5 (200 kb) (Fig. 3), using our new CRISPR-PCDup approach. We obtained $75 \mathrm{His}^{+}$ $\mathrm{Leu}^{+}$transformants using CRISPR-PCDup whereas no transformants were obtained using conventional PCDup (Table 3). Of the $75 \mathrm{His}^{+} \mathrm{Leu}^{+}$transformants obtained by CRISPR-PCDup, 25 were randomly selected and analyzed using PFGE and Southern blot analysis to verify whether or not the anticipated double duplication had occurred. Ten of the transformants showed double duplication as evidenced by the presence of a $200 \mathrm{~kb}$ and 160 $\mathrm{kb}$ band. Fig. $3 \mathrm{a}-\mathrm{c}$, show 10 out of 25 candidate transformants analyzed by PFGE and Southern blotting. Results shows that five out of 10 transformants had a double duplication. When we analyzed remaining 15 transformants by PFGE and Southern blotting, we found that five transformants had also undergone a double duplication while other 10 transformants had either a single duplication or no duplication event (data not shown). Therefore, we conclude that simultaneous double duplication is possible by using CRISPR-PCDup.

\section{Synthetic lethality is not caused by simultaneous double duplication}

After successfully duplicating two chromosomal regions simultaneously using CRISPR-PCDup technology, we next attempted to duplicate other chromosomal regions in two different chromosomes, namely Chr3-1 (158 kb) (1-158020) and Chr8-1 (200 kb) (1-202241). We used DNA modules harboring the CgLEU2 marker to duplicate the Chr3-1 region and the CgHIS3 marker to duplicate the Chr8-1 region. Leucine- and histidineminus (-Leu-His) medium was used to subsequently select $\mathrm{His}^{+} \mathrm{Leu}^{+}$transformants. $15 \mathrm{His}^{+} \mathrm{Leu}^{+}$transformants were obtained and these transformants were checked by colony PCR. None of the 15 transformants showed simultaneous duplication of the Chr3-1 and Chr8-1 regions. Next, another combination comprising the Chr3-1 and Chr14-4 (184 kb) (597394-784333) regions were chosen for simultaneous duplication

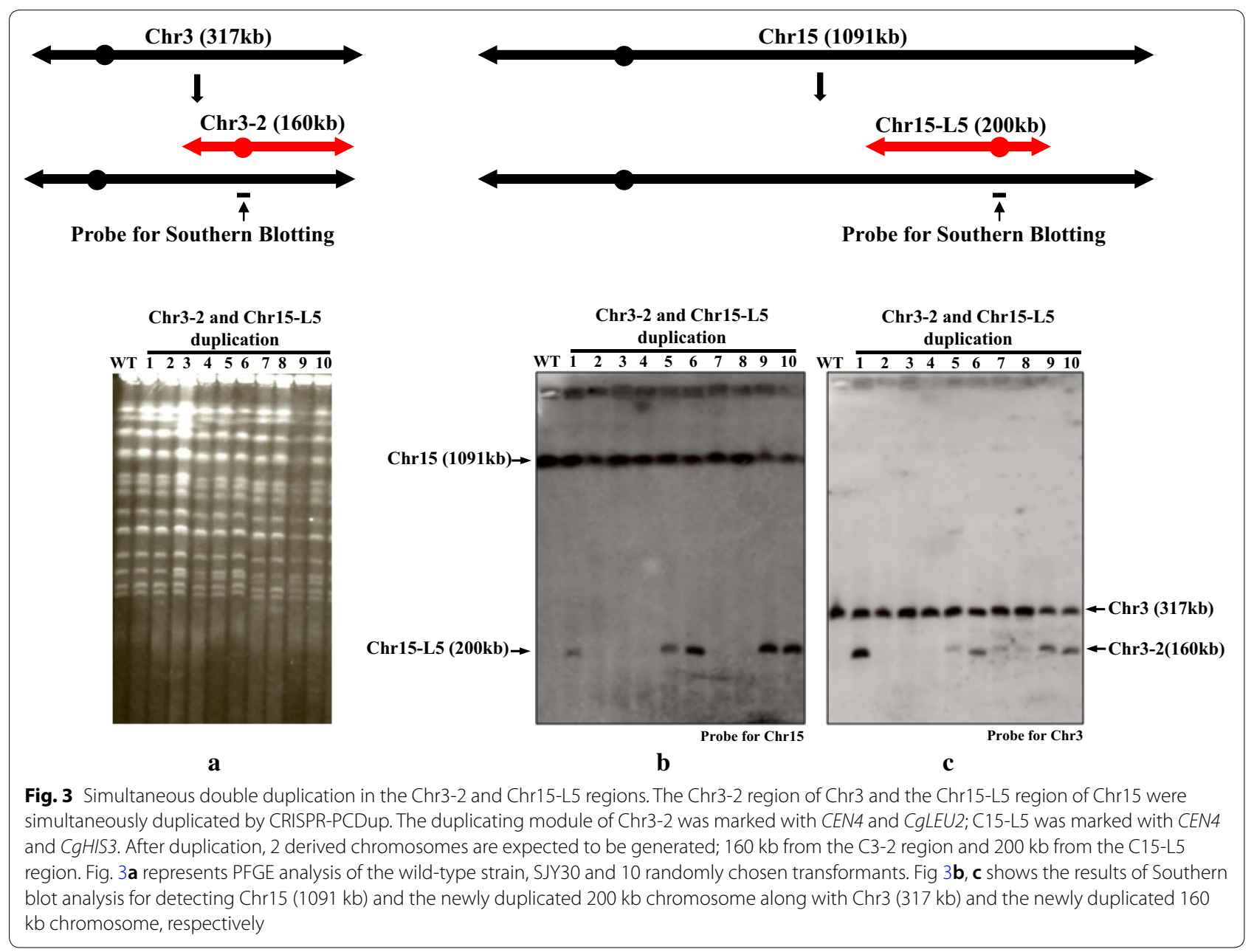




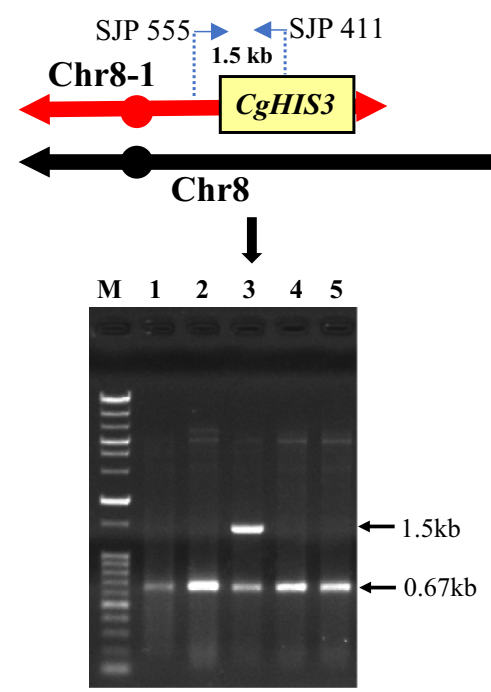

a
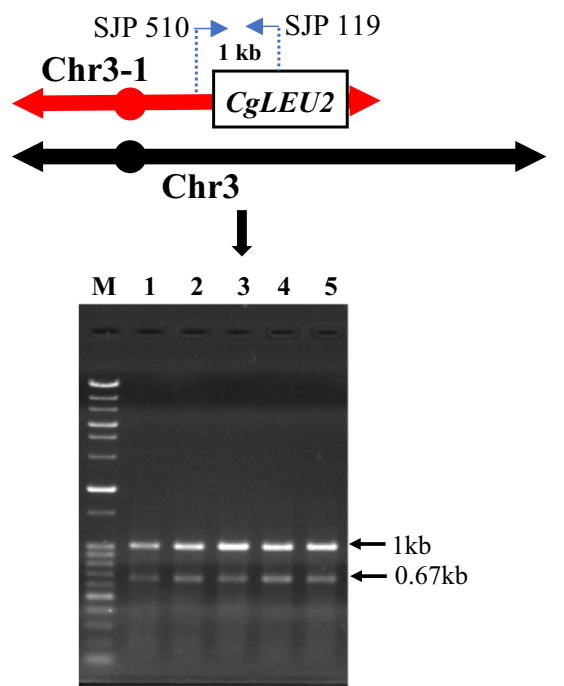

b
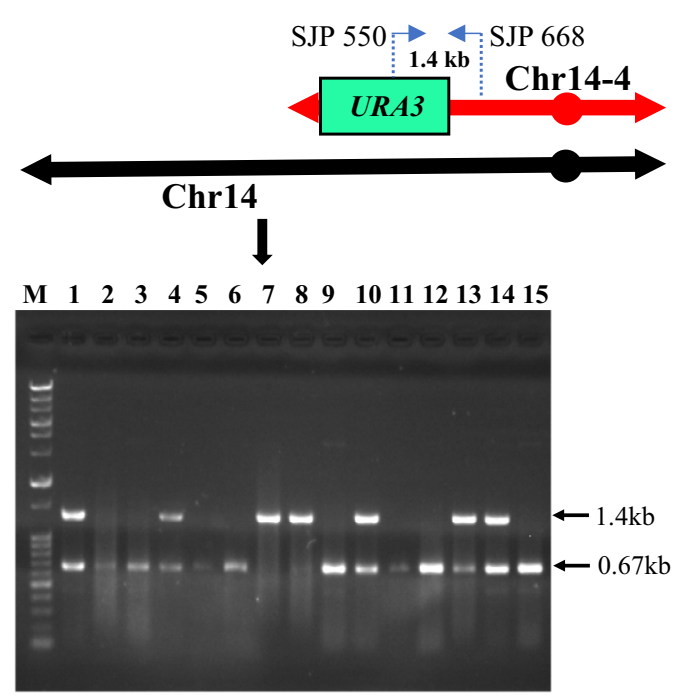

c
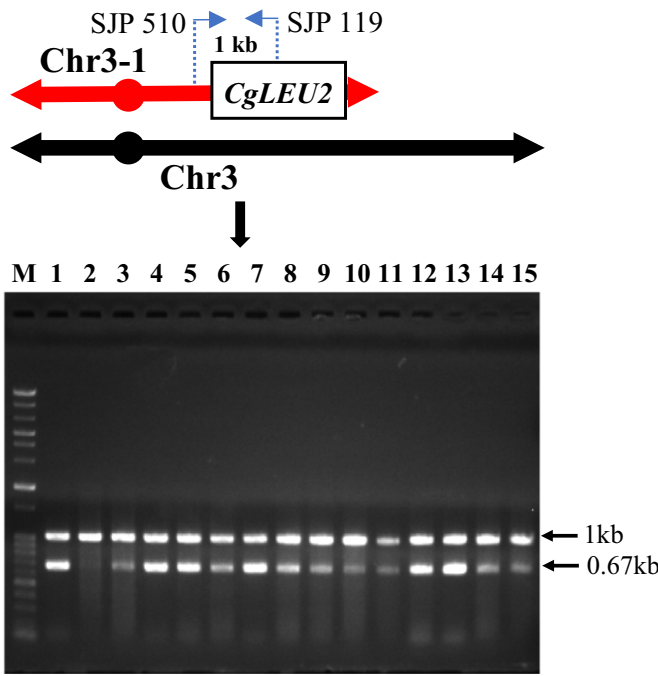

d

Fig. 4 Colony PCR analysis of Chr3-1 and Chr8-1 as well as Chr3-1 and Chr14-4 sequentially duplicated transformants. In the colony PCR, each lane represents independent transformants. Fig. $4 \mathbf{a}$, $\mathbf{b}$ represents the sequential duplication of the Chr3-1 and Chr8-1 regions, respectively. In Fig. 4a, primers SJP 555 and SJP 411 were used to amplify the $1.5 \mathrm{~kb}$ band from the duplicated Chr8-1 region and in Fig. 4b, primers SJP 510 and SJP 119 were used to amplify the $1 \mathrm{~kb}$ band from the duplicated Chr3-1 region. Fig. 4c , $\mathbf{d}$ represents the sequential duplication of the Chr3-1 and Chr14-4 regions, respectively. In Fig. 4c, primers SJP 550 and SJP 668 were used to amplify the $1.4 \mathrm{~kb}$ band from the duplicated Chr14-4 region and in Fig. 4d, primers SJP 510 and SJP 119 were used to amplify the $1 \mathrm{~kb}$ band from the duplicated Chr3-1 region. In all PCR analysis the $0.67 \mathrm{~kb}$ CNE1 gene on Chromosome 1 was also amplified as an internal control by a common set of primers SJP 121 and SJP 242

mediated by CRISPR-PCDup. We used DNA modules harboring the CgLEU2 marker to duplicate the Chr3-1 region and the URA3 marker to duplicate the Chr14-4 region. Leucine- and uracil- minus (-LeuUra) medium was used to select $\mathrm{Leu}^{+} \mathrm{Ura}^{+}$transformants. $66 \mathrm{Leu}^{+} \mathrm{Ura}^{+}$transformants were isolated and 15 transformants subsequently checked by colony PCR. The results revealed that none of the 15 transformants showed simultaneous duplication of the Chr3-1 and Chr14-4 regions.

Unsuccessful double duplication may be due to synthetic lethality caused by simultaneously duplicating these two sets of chromosomal regions. To investigate this possibility, we attempted to construct the double duplication in a sequential manner. Initially, the Chr3-1 region was duplicated and an attempt was made 
to duplicate the Chr8-1 region. In all, $155 \mathrm{His}^{+} \mathrm{Leu}^{+}$ transformants were obtained and 5 transformants were arbitrarily picked for colony PCR analysis. The colony PCR revealed that of the 5 transformants, 1 gave the expected result (Fig. 4a, b). We also attempted to sequentially duplicate the Chr14-4 region in the Chr3-1 duplicated transformants. In total, $796 \mathrm{Leu}^{+} \mathrm{Ura}^{+}$transformants were isolated and 15 were arbitrarily picked for colony PCR. Of these, 7 transformants had the expected double duplication (Fig. 4c, d). Primers used for colony PCR are listed in Additional file 1: Table S5.

These findings indicated that sequential double duplication of the Chr3-1 and Chr8-1 regions as well as the Chr3-1 and Chr14-4 regions is possible. We also used an alternative approach to further confirm synthetic lethality is not caused by simultaneous double duplication. Tetrad analysis of diploids was conducted to investigate whether double duplication causes synthetic lethality or not by mating transformants harboring two single duplicated regions. For this purpose, mating type $\alpha$ host SJY30 was chosen and the Chr3-1 region was duplicated using the CgLEU2 harboring DNA module. In a separate experiment, we took mating type a host SJY415 and duplicated the Chr5-3 (177 kb) region with the CgHIS3 harboring DNA module (data not shown). A diploid is then constructed by mating two transformants harboring either the Chr3-1 or Chr5-3 duplicated regions. After making diploids, tetrad analysis was performed. This analysis revealed that $\mathrm{Leu}^{+} \mathrm{His}^{+}$spores were viable, confirming that double duplication of these two regions is not lethal. Overall, these observations suggest that unsuccessful obtaining of double duplication is not due to synthetic lethality caused by double duplication. We will discuss possible reason for this observation in Discussion section.

\section{Upper size limit of duplication by CRISPR-PCDup}

We previously reported that up to $300 \mathrm{~kb}$ of chromosomal region could be duplicated by conventional PCDup (Natesuntorn et al. 2015). However, in this study using CRISPR-PCDup we successfully duplicated single chromosomal regions more efficiently than using the conventional PCDup procedure. Thus, we examined whether the upper size limit of the duplicated regions is increased using CRISPR-PCDup technology. For this purpose, we attempted to construct a series of segmentally duplicated chromosomes of increasing size $(200 \mathrm{~kb}, 250 \mathrm{~kb}, 300 \mathrm{~kb}$, $350 \mathrm{~kb}$ and $400 \mathrm{~kb}$ of Chr15), (Table 4). We found that all the regions could be duplicated (Fig. 5a, b). In the case of conventional PCDup, we did not get any transformants for the duplication of $200 \mathrm{~kb}$ to $400 \mathrm{~kb}$. Previously, Natesuntorn et al. (2015) was able to duplicate up to $300 \mathrm{~kb}$ using conventional PCDup by employing 400 bp homology sequence in the DNA module for homologous recombination. By contrast, in this study, we used a $50 \mathrm{bp}$ homology sequence in the DNA module along with CRISPR/Cas9. Despite this much shorter homology sequence, we achieved duplication of up to $400 \mathrm{~kb}$. We believe that introduction of a DSB mediated by CRISPR/ Cas9 enabled at least $400 \mathrm{~kb}$ duplication even when a relatively short $50 \mathrm{bp}$ homology sequence was employed. Thus, in the absence of CRISPR/Cas9, a 50 bp homology might be insufficient for successful homologous recombination to occur.

\section{Discussion}

In this study, we have developed a novel chromosome engineering technology by combining CRISPR/Cas9 system with our previously developed PCDup technology which we called CRISPR-PCDup. Since integration of CRISPR/Cas9 system into PCDup method may increase homologous recombination frequency, we expected that CRISPR-PCDup enables targeting multiple chromosomal regions to be duplicated by a single transformation. Previously, DiCarlo et al. (2013) reported that foreign donor DNA was integrated with nearly $100 \%$ frequency at the target site when a DSB is induced by CRISPR/Cas9 in S. cerevisiae. Indeed, in this study we found that duplication efficiency was increased approximately 25 to 30 fold when targeting a single site with the help of CRISPR/ Cas9 (Table 3). In addition, the proportion of transformants analyzed with the desired karyotype by conventional PCDup was $0 \%$. By contrast, $100 \%$ of randomly selected transformants obtained using the CRISPRPCDup method possessed the anticipated chromosomal changes (Table 3). Although this technology has not yet been tested for other chromosomal regions,

\begin{tabular}{lcrl}
$\begin{array}{l}\text { Table 4 CRISPR-PCDup } \\
\text { of chromosomal region }\end{array}$ & can & duplicate & up to $400 k b$ \\
\hline $\begin{array}{l}\text { Size } \\
\text { of the duplication } \\
\text { (Name of region) }\end{array}$ & gRNA & $\begin{array}{l}\text { No. } \\
\text { of transformants }\end{array}$ & $\begin{array}{l}\text { Proportion } \\
\text { of correct } \\
\text { transformants }\end{array}$ \\
\hline $400 \mathrm{~kb}$ & & & $20 \%(2 / 10)$ \\
(Chr15-L1) & - & 0 & - \\
$350 \mathrm{~kb}$ & + & 40 & $20 \%(2 / 10)$ \\
(Chr15-L2) & - & 0 & - \\
$300 \mathrm{~kb}$ & + & 733 & $3 \%(1 / 35)$ \\
(Chr15-L3) & - & 0 & - \\
$250 \mathrm{~kb}$ & + & 114 & $20 \%(2 / 10)$ \\
(Chr15-L4) & - & 0 & - \\
$200 \mathrm{~kb}$ & + & 120 & $90 \%(9 / 10)$ \\
(Chr15-L5) & - & 0 & - \\
\hline
\end{tabular}




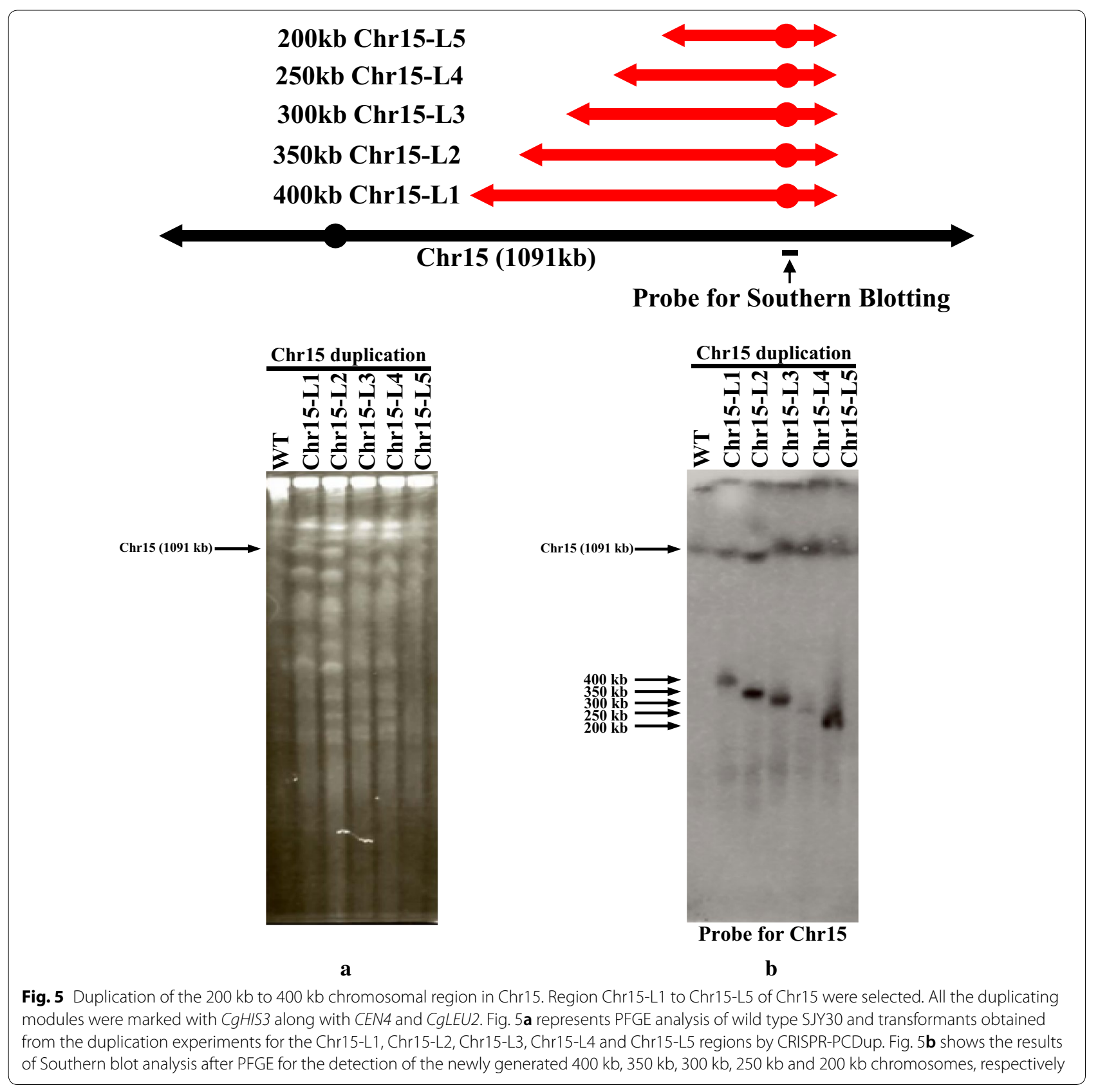

we suppose that CRISPR-PCDup may duplicate any chromosomal regions with significantly greater efficiency than conventional PCDup. Besides, a significant increase in the efficiency of a single duplication event is probably the reason for the success of simultaneous double duplications of at least two large chromosomal regions. We believe the enhanced efficiency of this new method arises from the DSBs induced by CRISPR/ Cas9 that stimulate an increased rate of homologous recombination.
Next, we attempted simultaneous duplication of two sets of chromosomal regions named as Chr3-1 and Chr8-1 as well as Chr3-1 and Chr14-4 but we did not get simultaneous double duplications in these two cases. Natesuntorn et al. (2015) proposed that duplicating chromosomal regions requires chromosome nondisjunction as one of possible mechanisms. It is likely that incorporation of CRISPR/Cas9 has influence on homologous recombination but not that on chromosome nondisjunction. Therefore, we think that the frequency of 
chromosome nondisjunction is the same even if we incorporated CRISPR/Cas9 system into our PCDup method. In this case, if the frequency of homologous recombination is not so high in two target sites, we may not get double duplication. By contrast, since sequential duplication needs only one homologous recombination for each transformation followed by possible chromosome nondisjunction, we think that this may be the reason by which we got duplication of Chr3-1 and Chr8-1 as well as Chr3-1 and Chr14-4 regions in a sequential manner but not simultaneously. On the other hand, we got success to duplicate Chr3-2 and Chr15-L5 regions simultaneously when we incorporated CRISPR/Cas9 system. Therefore, we think that possible reason for getting this success of obtaining double duplication simultaneously is that the frequency of homologous recombination became significantly higher by using CRISPR/Cas9 system compared with that of conventional PCDup method. Although it is not so easy to directly estimate the frequency of homologous recombination which is needed to duplicate multiple chromosomal regions simultaneously, we suggest that increased frequency of homologous recombination may contribute to the success of getting simultaneous duplication of Chr3-2 and Chr15-L5 regions.

In this study, we were able to lengthen the regions to be duplicated to $400 \mathrm{~kb}$ which is $100 \mathrm{~kb}$ larger than the longest duplication $(300 \mathrm{~kb})$ by conventional PCDup. In a previous study (Natesuntorn et al. 2015), we proposed that the upper-size limitation of chromosome duplication might be controlled by the frequency of chromosome nondisjunction because the rate of chromosome nondisjunction decreases as the length of the chromosome increases (Hieter et al. 1985). According to this data, larger duplicated chromosomes give rise to decreased rate of chromosome nondisjunction, but we believe that the number of resultant duplicated regions was increased significantly by CRISPR/Cas9 before chromosome nondisjunction occurs. As a result, chance of obtaining a longer duplicated chromosome might be increased and we think that this is the reason why we got viable transformants harboring $400 \mathrm{~kb}$ duplicated chromosome.

Simultaneous segmental duplication of multiple chromosomal regions is not reported in any organism. Here, we have demonstrated for the first time that it is possible to simultaneously duplicate two large segments of chromosomal regions (160 kb and $200 \mathrm{~kb}$ ) using our newly developed CRISPR-PCDup technology. Since segmental aneuploidy are occasionally found in industrial yeast strains displaying robustness (Borneman et al. 2011, Dunn et al. 2012) and we also previously revealed that duplicating several chromosomal regions gives rise to stress resistance against ethanol, high temperature, acetic and sulfuric acid (Natesuntorn et al. 2015), we think that CRISPR-PCDup technology should contribute to investigating combinatorial effect of segmental aneuploidy in an efficient way. Moreover, since duplicated chromosomes act as independent chromosomes comprising extra-copies of many genes, those chromosomes may be suitable for studying the effect of over-expression of many genes on cell physiology. In conclusion, CRISPR-PCDup is a promising tool not only for generating yeast strains that exhibit desired industrial traits but also for studying the fundamentals of genome function.

\section{Supplementary information}

\section{Supplementary information accompanies this paper at https://doi. org/10.1186/s13568-020-0957-4.}

Additional file 1: Table S1. Primers used for constructing DNA modules. Table S2. Primers used for constructing the gRNA expressing plasmid. Table S3. Primers used for Southern blotting. Table S4. Positions of duplication points on different chromosomes. Table S5. Primers used for colony PCR.

\section{Acknowledgements}

Not applicable.

\section{Authors' contributions}

$\mathrm{NH}, \mathrm{YS}$ and SH designed, performed the experiments and wrote the manuscript. SK and FE performed the experiments. KE, HT and SH designed and supervised the study. All the authors read and approved the final manuscript.

\section{Funding}

This work was supported by Japan Society for the Promotion of Science (JSPS) - KAKENHI, Grant-in-Aid for Scientific Research (B), Grant number [JP 15H04475] to S.H.

\section{Availability of data and materials}

The datasets generated during and/or analyzed during the current study are available from the corresponding author on reasonable request.

Ethics approval and consent to participate

This article does not contain any studies with human participants or animals performed by any of the authors.

\section{Consent for publication}

All authors gave their consent for publication.

\section{Competing interests}

The authors declare that they have no competing interests.

\section{Author details}

${ }^{1}$ Department of Applied Microbial Technology, Faculty of Biotechnology and Life Science, Sojo University, Ikeda 4-22-1, Nishi-ku, Kumamoto 860-0082, Japan. ${ }^{2}$ Department of Biotechnology, Graduate School of Engineering, Osaka University, Yamadaoka 2-1, Suita-shi, Osaka 565-0781, Japan.

Received: 23 November 2019 Accepted: 16 January 2020

Published online: 03 February 2020

\section{References}

Borneman AR, Desany BA, Riches D, Affourtit JP, Forgan AH, Pretorius IS, Egholm M, Chambers PJ (2011) Whole-genome comparison reveals novel 
genetic elements that characterize the genome of industrial strains of Saccharomyces cerevisiae. PLoS Genet 7(2):e1001287

Cong L, Ran FA, Cox D, Lin S, Barretto R, Habib N, Hsu PD, Wu X, Jiang W, Marraffini LA, Zhang $F$ (2013) Multiplex genome engineering using CRISPR/ Cas systems. Science 339:819-823

DiCarlo JE, Norville JE, Mali P, Rios X, Aach J, Church GM (2013) Genome engineering in Saccharomyces cerevisiae using CRISPR-Cas systems. Nucleic Acids Res 41:4336-4343

Dunn B, Richter C, Kvitek DJ, Pugh T, Sherlock G (2012) Analysis of the Saccharomyces cerevisiae pan-genome reveals a pool of copy number variants distributed in diverse yeast strains from differing industrial environments. Genome Res 22(5):908-924

Easmin F, Hassan N, Sasano Y, Ekino K, Taguchi H, Harashima S (2019a) gRNAtransient expression system for simplified gRNA delivery in CRISPR/Cas9 genome editing. J Biosci Bioeng 128(3):373-378

Easmin F, Sasano Y, Kimura S, Hassan N, Ekino K, Taguchi H, Harashima S (2019b) CRISPR-PCD and CRISPR-PCRep: Two novel technologies for simultaneous multiple segmental chromosomal deletion/replacement in Saccharomyces cerevisiae. J Biosci Bioeng. https://doi.org/10.1016/j.jbios c. 2019.08 .004

Gietz RD, Schiestl RH (2007) High-efficiency yeast transformation using the LiAc/SS carrier DNA/PEG method. Nat Protoc 2:31-34

Güldener U, Heck S, Fiedler T, Beinhauer J, Hegemann JH (1996) A new efficient gene disruption cassette for repeated use in budding yeast. Nucleic Acids Res 24:2519-2524

Hieter P, Mann C, Snyder M, Davis RW (1985) Mitotic stability of yeast chromosomes: a colony color assay that measures nondisjunction and chromosome loss. Cell 40:381-392

Jinek M, Chylinski K, Fonfara I, Hauer M, Doudna JA, Charpentier E (2012) A programmable dual-RNA-guided DNA endonuclease in adaptive bacterial immunity. Science 337:816-821
Natesuntorn W, Iwami K, Matsubara Y, Sasano Y, Sugiyama M, Kaneko Y, Harashima S (2015) Genome-wide construction of a series of designed segmental aneuploids in Saccharomyces cerevisiae. Sci Rep 5:12510 Sander JD, Joung JK (2014) CRISPR-Cas systems for editing, regulating and targeting genomes. Nat Biotechnol 32:347-355

Sasano Y, Nagasawa K, Kaboli S, Sugiyama M, Harashima S (2016) CRISPR-PCS: a powerful new approach to inducing multiple chromosome splitting in Saccharomyces cerevisiae. Sci Rep 6:30278

Storici F, Durham CL, Gordenin DA, Resnick MA (2003) Chromosomal sitespecific double-strand breaks are efficiently targeted for repair by oligonucleotides in yeast. Proc Natl Acad Sci U S A 100:14994-14999

Sugiyama M, Ikushima S, Nakazawa T, Kaneko Y, Harashima S (2005) PCRmediated repeated chromosome splitting in Saccharomyces cerevisiae. Biotechniques 38:909-914

Sugiyama M, Nakazawa T, Murakami K, Sumiya T, Nakamura A, Kaneko Y, Nishizawa M, Harashima S (2008) PCR-mediated one-step deletion of targeted chromosomal regions in haploid Saccharomyces cerevisiae. Appl Microbiol Biotechnol 80:545-553

Swinnen S, Thevelein JM, Nevoigt E (2012) Genetic mapping of quantitative phenotypic traits in Saccharomyces cerevisiae. FEMS Yeast Res 12:215-227

Winston F, Dollard C, Ricupero-Hovasse SL (1995) Construction of a set of convenient Saccharomyces cerevisiae strains that are isogenic to S288C. Yeast 11:53-55

\section{Publisher's Note}

Springer Nature remains neutral with regard to jurisdictional claims in published maps and institutional affiliations.

\section{Submit your manuscript to a SpringerOpen ${ }^{\odot}$ journal and benefit from:}

- Convenient online submission

- Rigorous peer review

- Open access: articles freely available online

- High visibility within the field

- Retaining the copyright to your article

Submit your next manuscript at $\boldsymbol{\nabla}$ springeropen.com 